\title{
Mathieu twining characters for K3
}

\section{Journal Article}

\section{Author(s):}

Gaberdiel, Matthias R.; Hohenegger, Stefan; Volpato, Roberto

Publication date:

2010-09

\section{Permanent link:}

https://doi.org/10.3929/ethz-b-000025519

\section{Rights / license:}

Creative Commons Attribution 4.0 International

Originally published in:

Journal of High Energy Physics 2010(9), https://doi.org/10.1007/JHEP09(2010)058 


\section{Mathieu twining characters for $\mathrm{K} 3$}

\section{Matthias R. Gaberdiel, Stefan Hohenegger and Roberto Volpato}

Institut für Theoretische Physik,

ETH Zurich, CH-8093 Zürich, Switzerland

E-mail: gaberdiel@itp.phys.ethz.ch, stefanh@itp.phys.ethz.ch, volpato@itp.phys.ethz.ch

ABSTRACT: The analogue of the McKay-Thompson series for the proposed Mathieu group action on the elliptic genus of K3 is analysed. The corresponding NS-sector twining characters have good modular properties and satisfy remarkable replication identities. These observations provide strong support for the conjecture that the elliptic genus of K3 carries indeed an action of the Mathieu group $\mathbb{M}_{24}$.

Keywords: Extended Supersymmetry, Conformal Field Models in String Theory

ArXiv EPrint: 1006.0221 


\section{Contents}

1 Introduction and summary 1

2 Elliptic genera and their modular properties 2

2.1 Symmetric powers 4

3 Mathieu symmetry and twining characters $\quad 6$

3.1 Explicit formulae for the twining characters $\quad 7$

$\begin{array}{ll}3.2 & \text { Replication formulae } \\ \end{array}$

4 Conclusions 13

$\begin{array}{ll}\text { A Definitions } & \mathbf{1 4}\end{array}$

$\begin{array}{ll}\text { A.1 McKay-Thompson series } & 15\end{array}$

$\begin{array}{lll}\text { A.2 Twining characters for K3 } & 15\end{array}$

\section{Introduction and summary}

Recently, Eguchi, Ooguri and Tachikawa [11] have observed that the elliptic genus of K3 seems to carry an action of the Mathieu group $\mathbb{M}_{24}$. More specifically, they noticed that the expansion coefficients of the elliptic genus of K3, when written in terms of the elliptic genera of $\mathcal{N}=4$ superconformal representations, can be expressed in terms of dimensions of Mathieu group representations. This intriguing observation is very reminiscent of the famous observation of McKay and Thompson who found that the Fourier expansion coefficients of the $J$-function can be written in terms of dimensions of representations of the Monster group. This led to a development that is now usually referred to as 'Monstrous Moonshine', see [18] for a nice review. One of the upshots of this analysis is that the $J$-function can be thought of as the partition function of a self-dual conformal field theory, the 'Monster conformal field theory' [3, 16], whose automorphism group is precisely the Monster group.

Unlike the situation of the Monster group, the dimensions of the Mathieu group are rather small, and thus there is a certain amount of arbitrariness in the above observation regarding the elliptic genus of K3. It would therefore be very interesting to subject this proposal to some more stringent tests. In the context of Monstrous Moonshine, one of the tests concerned the so-called McKay-Thompson series [22]. They are obtained from the $J$-function by replacing the dimensions of the Monster group representations by their characters with respect to a Monster group element. (In retrospect, they are the 'twining characters', where one inserts into the partition function of the Monster conformal field theory a Monster group element.) It was observed early on by Conway \& Norton that these 
McKay-Thompson series have nice modular properties and satisfy remarkable replication identities [5]. The former identities have a natural interpretation in terms of standard orbifold arguments [23], while the latter can be obtained by considering the twining characters for the symmetric power theories (as follows from our analysis in section 3.2).

In this paper we study the analogues of the McKay-Thompson series for the case of K3, i.e. the twining elliptic genera where we insert a Mathieu group element into the trace. Using the proposed decomposition of the coefficients of the elliptic genus in terms of Mathieu group representations, we can calculate the first few coefficients of these twining genera explicitly. As for the McKay-Thompson series, standard orbifold arguments suggest that they must have good modular properties. At least for the group elements of small order, these modular constraints are very constraining, and we can use them to determine the twining genera exactly. We furthermore show that they satisfy highly non-trivial replication identities, relating twining genera corresponding to different group elements to one another. The fact that all of this works out represents a very stringent consistency check for the proposal of [11]; indeed, our analysis implies that one of the identifications of the coefficients in terms of Mathieu representation dimensions must be different to what was proposed in [11]. Our analysis thus gives strong support to the idea that the elliptic genus of $\mathrm{K} 3$ carries indeed an action of the Mathieu group $\mathbb{M}_{24}$. It furthermore suggests that this is in some sense the natural supersymmetric generalisation of Monstrous Moonshine to the case of $\mathbb{M}_{24}$. (A different proposal for the analogue of Monstrous Moonshine for $\mathbb{M}_{24}$ was made some time ago in the context of self-dual bosonic conformal field theories by Dong \& Mason [8].)

The paper is organised as follows. In section 2 we recall some basic properties of elliptic genera and their modular properties. We also review how the elliptic genus of the symmetric power theory can be calculated in terms of the original elliptic genus. In section 3 we briefly describe the proposal of [11], and then calculate the twining elliptic genera and their associated NS twining characters. We explain in detail which modular properties the latter should possess. Using this insight we then identify the twining characters for group elements of small order in terms of known theta functions (or standard McKay-Thompson series), see (3.14). In section 3.2 we explain what replication formulae one should expect, and demonstrate in a large number of examples, that these identities are indeed satisfied for the twining characters of K3. Our conclusions and avenues for future research are described in section 4 . We have relegated some of the more technical material to an appendix.

\section{Elliptic genera and their modular properties}

Let us begin by reviewing some standard material about elliptic genera and their Jacobi and modular properties. The elliptic genus of an $\mathcal{N}=2$ superconformal algebra is defined by

$$
\phi(\tau, z)=\operatorname{Tr}_{\mathcal{H}_{R R}}\left(q^{L_{0}-\frac{c}{24}} e^{2 \pi i z J_{0}}(-1)^{F} \bar{q}^{\bar{L}_{0}-\frac{\bar{c}}{24}}(-1)^{\bar{F}}\right) .
$$

For the right-movers (whose modes are denoted by a bar), only the ground states contribute, and hence the above expression is in fact independent of $\bar{q}$. As is well-known [21], the modularity properties of conformal field theory together with spectral flow invariance and 
unitarity imply that the elliptic genus is a weak Jacobi form of index $m=\frac{c}{6}$ and weight zero [14]. A weak Jacobi form $\phi(\tau, z)$ of weight $w$ and index $m \in \mathbb{Z}$, with $(\tau, z) \in \mathbb{H} \times \mathbb{C}$, satisfies the transformation laws

$$
\begin{aligned}
\phi\left(\frac{a \tau+b}{c \tau+d}, \frac{z}{c \tau+d}\right) & =(c \tau+d)^{w} e^{2 \pi i m \frac{c z^{2}}{c \tau+d}} \phi(\tau, z) & \left(\begin{array}{ll}
a & b \\
c & d
\end{array}\right) \in \operatorname{SL}(2, \mathbb{Z}), \\
\phi\left(\tau, z+\ell \tau+\ell^{\prime}\right) & =e^{-2 \pi i m\left(\ell^{2} \tau+2 \ell z\right)} \phi(\tau, z) & \ell, \ell^{\prime} \in \mathbb{Z},
\end{aligned}
$$

and has a Fourier expansion

$$
\phi(\tau, z)=\sum_{n \geq 0, \ell \in \mathbb{Z}} c(n, \ell) q^{n} y^{\ell}
$$

with $c(n, \ell)=(-1)^{w} c(n,-\ell)$. For the case of $\mathrm{K} 3$ that will concern us primarily in this paper, the elliptic genus equals [10]

$$
\phi_{K 3}(\tau, z)=2 y+20+2 y^{-1}+q\left(20 y^{2}-128 y+216-128 y^{-1}+20 y^{-2}\right)+\mathcal{O}\left(q^{2}\right),
$$

where $y=e^{2 \pi i z}$. For the following it will be convenient to rescale the elliptic genus by a factor of $\frac{1}{2}$, i.e. to define

$$
\phi_{1 \mathrm{~A}}(\tau, z)=\frac{1}{2} \phi_{K 3}(\tau, z)=y+10+y^{-1}+q\left(10 y^{2}-64 y+108-64 y^{-1}+10 y^{-2}\right)+\mathcal{O}\left(q^{2}\right),
$$

which is then directly equal to the standard weak Jacobi form $\phi_{0,1}[14]$.

To understand the analogy with the bosonic Monster conformal field theory, it is useful to consider instead the NS-sector version of the elliptic genus, which is obtained by applying one half unit of spectral flow to the left-movers, i.e. $\ell=\ell^{\prime}=\frac{1}{2}$ in $(2.3)$; the shift by $\ell=\frac{1}{2}$ is familiar from the spectral flow automorphism

$$
L_{0} \mapsto L_{0}+\ell J_{0}+\ell^{2} \frac{c}{6}, \quad J_{0} \mapsto J_{0}+\ell \frac{c}{3},
$$

while the shift by $\ell^{\prime}=\frac{1}{2}$ is required so as to remove the $(-1)^{F}$ from the trace, in order to get the standard NS-sector partition function without an insertion of $(-1)^{F}$. The resulting NS-sector partition function will always be denoted by

$$
\chi(\tau, z)=\exp \left[2 \pi i m\left(\frac{\tau}{4}+z+\frac{1}{2}\right)\right] \phi\left(\tau, z+\frac{\tau}{2}+\frac{1}{2}\right) .
$$

Using the transformation properties of a Jacobi form it follows easily that

$$
\begin{aligned}
\chi(-1 / \tau, z / \tau) & =(-1)^{m} e^{2 \pi i \frac{m z^{2}}{\tau}} \chi(\tau, z) \\
\chi(\tau+2, z) & =(-1)^{m} \chi(\tau, z) \\
\chi\left(\tau, z+\ell \tau+\ell^{\prime}\right) & =e^{-2 \pi i m\left(\ell^{2} \tau+2 \ell z\right)} \chi(\tau, z) \quad \ell, \ell^{\prime} \in \mathbb{Z} .
\end{aligned}
$$

In order to obtain a standard modular form (rather than a Jacobi form) we may then put $z=0$; the resulting function (which we shall simply denote by $\chi(\tau)$ ) is non-trivial, and it 
has simple transformation laws under the congruence subgroup $\Gamma_{\theta}=\left\langle T^{2}, S\right\rangle$. For $m$ even we have a strict modular function and for $m$ odd we have a function with multiplier system given by -1 on the two generators [17],

$$
\chi(-1 / \tau)=(-1)^{m} \chi(\tau), \quad \chi(\tau+2)=(-1)^{m} \chi(\tau) .
$$

For the case of K3 it is explicitly given as [17]

$$
\chi_{1 \mathrm{~A}}(\tau)=\frac{1}{2} \chi_{K 3}(\tau)=\left(\frac{2 \vartheta_{4}(\tau)}{\vartheta_{2}(\tau)}\right)^{2}-\left(\frac{2 \vartheta_{2}(\tau)}{\vartheta_{4}(\tau)}\right)^{2}=q^{-\frac{1}{4}}\left(1-20 q^{\frac{1}{2}}+\cdots\right) .
$$

Our conventions regarding the theta functions are described in the appendix.

It should be noted that specialising to $z=0$ in the NS-sector does not loose any information. Indeed, the NS character $\chi_{1 \mathrm{~A}}(\tau)$ determines $\phi_{1 \mathrm{~A}}\left(\tau, \frac{1}{2}\right)$ by setting $z=0$ in

$$
\chi_{1 \mathrm{~A}}(\tau, z)=\phi_{1 \mathrm{~A}}(\tau, 0) \frac{\vartheta_{4}(\tau, z)^{2}}{\vartheta_{2}(\tau, 0)^{2}}-\phi_{1 \mathrm{~A}}\left(\tau, \frac{1}{2}\right) \frac{\vartheta_{3}(\tau, z)^{2}}{\vartheta_{2}(\tau, 0)^{2}},
$$

and using that the coefficient of the first term is simply the Witten index $\phi_{1 \mathrm{~A}}(\tau, 0)=12$. On the other hand, this information determines the full elliptic genus via

$$
\phi_{1 \mathrm{~A}}(\tau, z)=\phi_{1 \mathrm{~A}}(\tau, 0) \frac{\vartheta_{2}(\tau, z)^{2}}{\vartheta_{2}(\tau, 0)^{2}}+\phi_{1 \mathrm{~A}}\left(\tau, \frac{1}{2}\right) \frac{\vartheta_{1}(\tau, z)^{2}}{\vartheta_{2}(\tau, 0)^{2}} .
$$

Thus the knowledge of $\chi_{1 \mathrm{~A}}(\tau)$ is equivalent to that of $\phi_{1 \mathrm{~A}}(\tau, z)$. The advantage of working with $\chi_{1 \mathrm{~A}}(\tau)$ is that it is the natural supersymmetric analogue of the partition function of a bosonic conformal field theory for which Monstrous Moonshine was originally formulated.

\subsection{Symmetric powers}

For the following it will also be important to consider the symmetric powers of the K3 conformal field theory. Given any $\mathcal{N}=2$ superconformal field theory $\mathcal{H}$, we can consider the symmetric power theory

$$
\mathcal{H}^{(p)}=\underbrace{(\mathcal{H} \otimes \cdots \otimes \mathcal{H})}_{\mathrm{p} \text { times }} / \mathbb{Z}_{p}
$$

where the generator $\pi$ of $\mathbb{Z}_{p}$ is the cyclic permutation of length $p$. We shall concentrate in the following on the case when $p$ is prime; then all elements of $\mathbb{Z}_{p}$ are cyclic permutations of cycle length $p$. Given the elliptic genus of the original $\mathcal{H}$ theory, we can then determine the elliptic genus of $\mathcal{H}^{(p)}$, along the lines described in [6]. ${ }^{1}$ From the untwisted sector of the $\mathbb{Z}_{p}$ orbifold we get the contribution

$$
\phi_{U}^{(p)}(\tau, z)=\frac{1}{p}\left((\phi(\tau, z))^{p}+(p-1) \phi(p \tau, p z)\right) .
$$

\footnotetext{
${ }^{1}$ The final result of $[6]$ is the elliptic genus of the orbifold $(\mathcal{H} \otimes \cdots \otimes \mathcal{H}) / S_{p}$, where $S_{p}$ is the whole permutation group of $p$ symbols. However, analogous methods can be applied to the case of orbifolds by cyclic subgroups $\mathbb{Z}_{p} \subset S_{p}$.
} 
Here the first term is the elliptic genus without the insertion of any orbifold generator, while the second term gives the contributions coming from $\pi^{l}$ with $l=1, \ldots, p-1$. Since each such $\pi^{l}$ is a cyclic permutation of order $p$, only the states of the form $(u \otimes u \otimes \cdots \otimes u)$ contribute, and this leads to $\phi(p \tau, p z)$ for each of these terms.

There are $(p-1)$ twisted sectors, and each of them contributes the same, thus giving for the whole twisted sector

$$
\phi_{T}^{(p)}(\tau, z)=\frac{(p-1)}{p} \sum_{l=0}^{p-1} \phi\left(\frac{\tau+l}{p}, z\right),
$$

where the sum over $l$ implements the orbifold projection in the twisted sector. Thus the elliptic genus of the symmetric power theory is (for $p$ prime)

$$
\phi^{(p)}(\tau, z)=\frac{1}{p}(\phi(\tau, z))^{p}+\frac{(p-1)}{p} H_{p} \phi(\tau, z),
$$

where $H_{p}$ denotes the action of the Hecke operator

$$
H_{p} \phi(\tau, z)=\phi(p \tau, p z)+\sum_{l=0}^{p-1} \phi\left(\frac{\tau+l}{p}, z\right) .
$$

It is well known that (2.17) is again a weak Jacobi form of index $m p$ provided that $\phi(\tau, z)$ has index $m$. For example, this follows directly from the fact that the Hecke operator (2.18) maps weak Jacobi forms to weak Jacobi forms, see [14].

For our purposes, it is also convenient to rewrite (2.18) for the NS-sector character $\chi^{(p)}(\tau)$. The result depends on whether $p=2$ or $p$ odd. For $p=2$ we find

$$
\chi^{(2)}(\tau)=\chi\left(2 \tau, \frac{1}{2}\right)+\phi\left(\frac{\tau}{2}, \frac{1}{2}\right)+(-1)^{m} \phi\left(\frac{\tau}{2}+\frac{1}{2}, 0\right)
$$

where $\chi(\tau, z)$ is the NS-sector function defined in (2.8), while $\phi(\tau, z)$ is the elliptic genus in the R-sector. In particular, the last expression is therefore actually a constant since $\phi(\tau, 0)$ is the Witten index. The emergence of the R-sector as the 'twisted' sector of the NS-sector is familiar from standard $\mathbb{Z}_{2}$ orbifold considerations. For the case of the K3 character the relevant formula is ${ }^{2}$

$$
\begin{aligned}
\chi_{1 \mathrm{~A}}^{(2)}(\tau) & =\left[\left(\frac{2 \vartheta_{3}(2 \tau)}{\vartheta_{2}(2 \tau)}\right)^{2}-\left(\frac{2 \vartheta_{2}(2 \tau)}{\vartheta_{3}(2 \tau)}\right)^{2}\right]+\left[\left(\frac{2 \vartheta_{3}\left(\frac{\tau}{2}\right)}{\vartheta_{4}\left(\frac{\tau}{2}\right)}\right)^{2}-\left(\frac{2 \vartheta_{4}\left(\frac{\tau}{2}\right)}{\vartheta_{3}\left(\frac{\tau}{2}\right)}\right)^{2}\right]-12 \\
& =\chi_{1 \mathrm{~A}}(\tau)^{2}+36
\end{aligned}
$$

The fact that the left-hand side can be expressed in terms of a polynomial of the original $\mathrm{K} 3$ character is a consequence of $\chi_{1 \mathrm{~A}}(\tau)^{2}$ being a Hauptmodul (without any multiplier system) for the congruence subgroup $\Gamma_{\theta}$. Since the left-hand is a modular form for $\Gamma_{\theta}$ (without any multiplier system), it must therefore be a polynomial of $\chi_{1 A}(\tau)^{2}$.

\footnotetext{
${ }^{2}$ We shall state these and any following formulae for the rescaled elliptic genera, see (2.6) and (2.11).
} 
For $p>2$ (recall that $p$ is prime, and therefore now odd) the formula is instead

$$
\chi^{(p)}(\tau)=\chi(p \tau)+\sum_{l=0}^{p-1} \chi\left(\frac{\tau+4 l}{p}\right), \quad p \geq 3 \text { prime. }
$$

For the case of the K3 character we then find, for example for $p=3$,

$$
\begin{aligned}
\chi_{1 \mathrm{~A}}^{(3)}(\tau) & =\chi_{1 \mathrm{~A}}(3 \tau)+\chi_{1 \mathrm{~A}}\left(\frac{\tau}{3}\right)+\chi_{1 \mathrm{~A}}\left(\frac{\tau+4}{3}\right)+\chi_{1 \mathrm{~A}}\left(\frac{\tau+8}{3}\right) \\
& =\chi_{1 \mathrm{~A}}(\tau)^{3}+60 \chi_{1 \mathrm{~A}}(\tau)
\end{aligned}
$$

By the same token as above, this formula follows from the fact that the left-hand side is again a modular form (with multiplier system -1 on $S$ and $T^{2}$ ) for $\Gamma_{\theta}$, and that the Hauptmodul for these is $\chi_{1 \mathrm{~A}}(\tau)$. Because of the non-trivial multiplier system of the lefthand-side the expression now only involves odd powers of $\chi_{1 \mathrm{~A}}(\tau)$.

We should note that these relations are the natural analogue of the replication formulae for the $J$-function, which read for $p=2$ and $p=3$

$$
\begin{gathered}
J(2 \tau)+J\left(\frac{\tau}{2}\right)+J\left(\frac{\tau+1}{2}\right)=J^{2}(\tau)-2 a_{1} \\
J(3 \tau)+J\left(\frac{\tau}{3}\right)+J\left(\frac{\tau+1}{3}\right)+J\left(\frac{\tau+2}{3}\right)=J^{3}(\tau)-3 a_{1} J(\tau)-3 a_{2} .
\end{gathered}
$$

Here $J(\tau)$ is the famous $J$-function

$$
J(\tau)=\sum_{k=-1}^{\infty} a_{k} q^{k}=q^{-1}+196884 q+21493760 q^{2}+\mathcal{O}\left(q^{3}\right),
$$

that plays a central role in Monstrous moonshine, see [18] for a nice review.

\section{Mathieu symmetry and twining characters}

The elliptic genus of K3 can be thought of as the partition function of the $\mathcal{N}=2$ half-BPS states of type II string theory on K3. It is suggested by the work of [11] that the space of these half-BPS states carries an action of the Mathieu group $\mathbb{M}_{24}$. Indeed, it was found in [11], following on from earlier work [9], that the elliptic genus of K3 can be written as

$$
\phi_{1 \mathrm{~A}}(\tau, z)=24 \operatorname{ch}_{h=\frac{1}{4}, l=0}^{\mathcal{N}=4}(\tau, z)+\sum_{n=0}^{\infty} A_{n} \operatorname{ch}_{h=n+\frac{1}{4}, l=\frac{1}{2}}^{\mathcal{N}=4}(\tau, z),
$$

where $\operatorname{ch}_{h=\frac{1}{4}, l=0}^{\mathcal{N}=4}$ is the elliptic genus of the short $\mathcal{N}=4$ representation with $h=\frac{1}{4}$ and $l=0-$ see $[12,13]$ for an explicit formula — while

$$
\operatorname{ch}_{h, l=\frac{1}{2}}^{\mathcal{N}=4}(\tau, z)=q^{h-\frac{3}{8}} \frac{\vartheta_{1}(\tau, z)^{2}}{\eta(\tau)^{3}}
$$


is the elliptic genus of a long $\mathcal{N}=4$ representation. ${ }^{3}$ The observation of [11] was that the coefficients $A_{n}$ can be written in terms of dimensions of irreducible representations of the Mathieu group $\mathbb{M}_{24}$. (The character table of $\mathbb{M}_{24}$, from which one can read off in particular the dimensions of the irreducible representations, is given in the appendix.) More explicitly,

$$
\begin{aligned}
24 & =\mathbf{2 3}+\mathbf{1} & -A_{0}=2 & =\mathbf{1}+\mathbf{1} \\
A_{1}=90 & =\mathbf{4 5}+\overline{\mathbf{4 5}} & A_{2}=462 & =\mathbf{2 3 1}+\overline{\mathbf{2 3 1}} \\
A_{3}=1540 & =\mathbf{7 7 0}+\overline{\mathbf{7 7 0}} & A_{4}=4554 & =\mathbf{2 2 7 7}+\overline{\mathbf{2 2 7 7}} \\
A_{5}=11592 & =2 \cdot \mathbf{5 7 9 6} & A_{6}=27830 & =2 \cdot \mathbf{3 5 2 0}+2 \cdot \mathbf{1 0 3 9 5}
\end{aligned}
$$

and

$$
A_{7}=61686=2 \cdot \mathbf{1 7 7 1}+2 \cdot \mathbf{2 0 2 4}+2 \cdot \mathbf{5 3 1 3}+2 \cdot \mathbf{5 7 9 6}+2 \cdot \mathbf{5 5 4 4}+2 \cdot \mathbf{1 0 3 9 5} .
$$

Note that, relative to [11], we have absorbed the prefactor of 2 in [11, eq. (1.11)] into the definition of $A_{n}$. Then we can write the $A_{n}$ in terms of real representations, i.e. sums of representations and their conjugate representations. Furthermore, for reasons that will be explained momentarily, our expression for $A_{7}$ differs from the decomposition suggested in [11]. We should mention though that while $A_{7}$ given in (3.4) is consistent with what is done below, it is not uniquely specified by this.

\subsection{Explicit formulae for the twining characters}

Obviously, as is apparent from the last comment, there is much arbitrariness in these decompositions, and they are only a suggestive hint of some underlying deeper structure. The main aim of this paper is to subject this proposal to a much more refined test. To this end we shall study the corresponding 'twining genus', i.e. the analogues of the McKay-Thompson series in the case of Monstrous Moonshine. This is to say, we shall calculate not just the elliptic genus itself, but the elliptic genus with the insertion of a group element $g \in \mathbb{M}_{24}$

$$
\phi_{g}(\tau, z)=\operatorname{Tr}_{\mathcal{H}_{R R}}\left(g q^{L_{0}-\frac{c}{24}} e^{2 \pi i z J_{0}}(-1)^{F} \bar{q}^{\bar{L}_{0}-\frac{\bar{c}}{24}}(-1)^{\bar{F}}\right) .
$$

Technically speaking, the calculation of $\phi_{g}$ differs from $\phi$ by replacing $A_{n}$ in (3.1) by the characters of $g$ in the representations described in (3.3). Obviously, the character of $g$ only depends on its conjugacy class, and thus all the information we need is contained in the character table of $\mathbb{M}_{24}$ that is included in the appendix.

One immediate problem with this approach is that we only know how to write $A_{n}$ in terms of dimensions of representations of $\mathbb{M}_{24}$ for the first few values of $n$. We can therefore only directly determine the low powers of $q$ of the twining genera $\phi_{g}$. If this was all there was to it, this would not be particularly interesting, and it would certainly not lead to any non-trivial consistency check of the proposal. The reason why interesting information can be obtained in this manner is that the twining genera must have fairly specific modular

\footnotetext{
${ }^{3}$ Strictly speaking, the $\mathcal{N}=4$ representation with $n=0\left(h=\frac{1}{4}\right)$ is short, and thus (3.2) for $h=\frac{1}{4}$ is not the elliptic genus of a single representation, but rather involves a sum of representations.
} 
properties. One way to see this explicitly is to translate the R-sector series $\phi_{g}(\tau, z)$ into the NS-sector as in (2.8), and set $z=0$. The resulting expression is the NS-sector twining character $\chi_{g}(\tau)$, i.e. the character in the NS-sector with the insertion of the Mathieu group element $g$. Note that by the same arguments as above we are not loosing any information in doing so, because the analogues of (2.12) and (2.13) are

$$
\chi_{g}(\tau, z)=\phi_{g}(\tau, 0) \frac{\vartheta_{4}(\tau, z)^{2}}{\vartheta_{2}(\tau, 0)^{2}}-\phi_{g}\left(\tau, \frac{1}{2}\right) \frac{\vartheta_{3}(\tau, z)^{2}}{\vartheta_{2}(\tau, 0)^{2}},
$$

and

$$
\phi_{g}(\tau, z)=\phi_{g}(\tau, 0) \frac{\vartheta_{2}(\tau, z)^{2}}{\vartheta_{2}(\tau, 0)^{2}}+\phi_{g}\left(\tau, \frac{1}{2}\right) \frac{\vartheta_{1}(\tau, z)^{2}}{\vartheta_{2}(\tau, 0)^{2}}
$$

where now the constant term equals

$$
\phi_{g}(\tau, 0)=\frac{1}{2} \operatorname{Tr}_{\mathbf{2 3} \oplus \mathbf{1}}(g) .
$$

Thus we can reconstruct the full twining genus $\phi_{g}(\tau, z)$ from the twining character $\chi_{g}(\tau)$, and vice versa. In terms of the usual box notation, $\chi_{g}(\tau)$ corresponds to

$$
\chi_{g}(\tau) \longleftrightarrow(-1, \mathbf{1}) \underset{(-1, \mathbf{g})}{\square}
$$

where the ' -1 's indicate that the trace is taken in the NS-sector, and without any insertion of $(-1)^{F}$, respectively. Under a modular transformation it is believed that these twining and twisted characters transform as

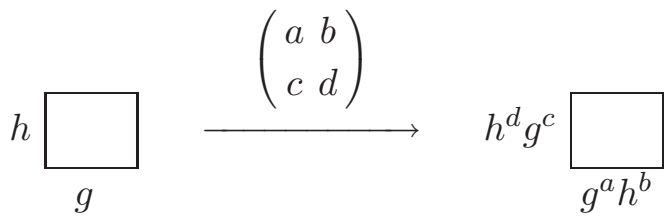

For the case at hand, it therefore follows that the twining character corresponding to (3.9) is invariant (possibly up to some phase) under a modular transformation provided that

$$
a+b, c+d \in 2 \mathbb{Z}+1, \quad c=0 \quad \bmod o(g),
$$

where $o(g)$ is the order of the group element $g$. [Here we have used that the group operation is $\left(\epsilon_{1}, g_{1}\right) \cdot\left(\epsilon_{2}, g_{2}\right)=\left(\epsilon_{1} \epsilon_{2}, g_{1} \cdot g_{2}\right)$, where $\epsilon_{j} \in\{ \pm 1\}$ and $g_{i} \in \mathbb{M}_{24}$. We have furthermore used that the conditions in (3.11) together with the requirement that $a d-b c=1$ imply that $(a, o(g))=1$. For the Mathieu group $\mathbb{M}_{24}$ it then follows that $g^{a}$ is in the same conjugacy class as $g$.] The subgroup of $\mathrm{SL}(2, \mathbb{Z})$ that is characterised by (3.11) contains in particular

$$
\Gamma^{g}=\Gamma_{\theta} \cap \Gamma_{0}(o(g)),
$$

where $\Gamma_{\theta}$ was introduced before, and

$$
\Gamma_{0}(N)=\left\{\left(\begin{array}{ll}
a & b \\
c & d
\end{array}\right) \in \operatorname{SL}(2, \mathbb{Z}): c=0 \bmod (N)\right\} .
$$


The property of a function to be modular invariant under $\Gamma^{g}$ (possibly up to some multiplier system) is a very strong condition, and knowing the first few terms usually determines the function uniquely.

Thus the non-trivial consistency test we will be performing proceeds as follows. Using the low-lying decomposition (3.3), we can calculate the first few coefficients of the twining characters $\chi_{g}(\tau)$ explicitly. On the other hand, we know that these functions must be invariant under $\Gamma^{g}$, and thus there is the highly non-trivial consistency condition whether this ansatz can be completed in a $\Gamma^{g}$-invariant manner. The results we find are consistent with $A_{n}$ given in (3.3) and (3.4), but not with the formula for $A_{7}$ suggested in [11]. They represent a highly non-trivial consistency condition for the conjecture of [11] that the elliptic genus of K3 carries an action by the Mathieu group $\mathbb{M}_{24}$.

The constraints coming from the modular properties are tighter the larger $\Gamma^{g}$ is, i.e. the smaller the order of the group element is. In particular, we should therefore expect to have good control over the twining characters corresponding to group elements of small order. Indeed, this turns out to be correct, and we have even found closed form expressions that match with the low lying coefficients and have the correct modular properties. More specifically, we find that - in the following the label refers to the conjugacy class of the Mathieu group element that is described in the appendix

$$
\begin{aligned}
& \chi_{1 \mathrm{~A}}(\tau)=T_{[8 \mathrm{a}]}\left(\frac{\tau}{4}\right) \quad \text { - see eq. }(2.11) \\
& \chi_{2 \mathrm{~A}}(\tau)=4 \frac{\vartheta_{4}(\tau)^{2}}{\vartheta_{2}(\tau)^{2}} \simeq T_{[16 \mathrm{~b}]}\left(\frac{\tau}{4}\right) \\
& \chi_{2 \mathrm{~B}}(\tau)=4 \frac{\vartheta_{3}(\tau)^{2}}{\vartheta_{2}(\tau)^{2}} \simeq T_{[16 \mathrm{~b}]}\left(\frac{\tau}{4}\right) \\
& \chi_{3 \mathrm{~A}}(\tau)=\left(\frac{\eta(\tau)}{\eta(3 \tau)}\right)^{3} \frac{\vartheta_{3}(3 \tau)}{\vartheta_{3}(\tau)}=T_{[24 \mathrm{c}]}\left(\frac{\tau}{4}\right) \\
& \chi_{4 \mathrm{~A}}(\tau)=\left(\frac{\eta(\tau) \eta(2 \tau)}{\eta\left(\frac{\tau}{2}\right) \eta(4 \tau)}\right)^{4}=T_{[16 \mathrm{~A}]}\left(\frac{\tau}{4}\right) \\
& \chi_{4 \mathrm{~B}}(\tau)=2 \frac{\vartheta_{3}(2 \tau)}{\vartheta_{2}(2 \tau)}=T_{[16 \mathrm{~B}]}\left(\frac{\tau}{4}\right) \\
& \chi_{4 \mathrm{C}}(\tau)=4 \frac{\vartheta_{3}(\tau)^{2} \vartheta_{3}(2 \tau)}{\vartheta_{2}(\tau)^{2} \vartheta_{4}(2 \tau)} \\
& \chi_{5 \mathrm{~A}}(\tau)=q^{-\frac{1}{4}}\left(1+3 q+4 q^{\frac{3}{2}}+4 q^{2}+4 q^{\frac{5}{2}}+\cdots\right)=T_{[40 \mathrm{a}]}\left(\frac{\tau}{4}\right) \\
& \chi_{6 \mathrm{~A}}(\tau)=\left(\frac{\eta\left(\frac{3}{2} \tau\right) \eta(2 \tau)}{\eta\left(\frac{\tau}{2}\right) \eta(6 \tau)}\right)^{2}=T_{[24 \mathrm{H}]}\left(\frac{\tau}{4}\right) .
\end{aligned}
$$

Our conventions for the Jacobi theta functions, and the first few coefficients of the power series expansions of the relevant McKay-Thompson series are given in the appendix. We 
have again normalised these functions so that they start with $1 \cdot q^{-\frac{1}{4}}$, compare $(2.11)$. In most cases, the functions can be identified with a standard McKay-Thompson series $T_{[*]}$ (evaluated at $\frac{\tau}{4}$ ) - if this is the case the corresponding identity is given above. For the conjugacy class $2 \mathrm{~A}$ and $2 \mathrm{~B}$ the coefficients only agree with the McKay-Thompson series up to some signs; this is indicated by $\simeq$. In all cases we have explicitly checked that these functions are invariant under $\Gamma^{g}$ in (3.12) up to some multiplier system. The multiplier system is non-trivial in all cases since all twining characters transform as -1 under $T^{2} \in \Gamma^{g}$. In addition, $\chi_{1 \mathrm{~A}}$ transforms as -1 under $S$ (compare (2.9)), while $\chi_{4 \mathrm{C}}$ transforms as $-i$ under $S T^{4} S$. (We have not checked the precise form of the multiplier system for the class corresponding to $5 \mathrm{~A}$.)

The attentive reader will notice that (3.14) accounts for all twining characters of group elements up to order 6 , except for the ones corresponding to $3 \mathrm{~B}$ and $6 \mathrm{~B}$. While we have not managed to find any closed formulae for them - explicit formulae for the first few coefficients of their power series expansion are given in the appendix — we have an indirect argument that suggests that at least the twining character corresponding to $3 \mathrm{~B}$ has good modular properties; this will be explained in the following section. We should also mention that some of these conjugacy classes are actually contained in $\mathbb{M}_{23}$ - these are the conjugacy classes $1 \mathrm{~A}, 2 \mathrm{~A}, 3 \mathrm{~A}, 4 \mathrm{~B}, 5 \mathrm{~A}, 6 \mathrm{~A}$ - while the others $(2 \mathrm{~B}, 3 \mathrm{~B}, 4 \mathrm{~A}, 4 \mathrm{C})$ lie only in $\mathbb{M}_{24}$ but not in $\mathbb{M}_{23}$. These latter classes are characterised by the property that $\operatorname{Tr}_{\mathbf{2 3} \oplus \mathbf{1}}(g)=0$. For them the relation between the twining characters and the twining genera (see (3.6) and (3.7)) is therefore particularly simple, and we have

$$
\phi_{g}(\tau, z)=-\chi_{g}(\tau) \frac{\vartheta_{1}(\tau, z)^{2}}{\vartheta_{3}(\tau, 0)^{2}} \quad \text { if } \operatorname{Tr}_{\mathbf{2 3} \oplus \mathbf{1}}(g)=0 .
$$

Thus for the elements in $\mathbb{M}_{24}$ that are not in $\mathbb{M}_{23}$, the modular properties of $\phi_{g}(\tau, z)$ are a direct consequence of those of $\chi_{g}(\tau)$. However, apart from this simplification, we do not see any difference between the behaviour of the elements that lie in $\mathbb{M}_{23}$ and those that do not.

\subsection{Replication formulae}

One of the remarkable properties of the McKay-Thompson series in Monstrous Moonshine are their replication relations. They are a direct generalisation of the $J$-function replication identities (2.23) to the McKay-Thompson series. In the following we want to give a conformal field theoretic argument that suggests that similar relations should also hold for the twining characters of K3, and more specifically, what precise form they should take. Incidentally, if we applied the following argument to the Monster theory it would give a physics explanation of the replication identities of Monstrous Moonshine.

The idea of the argument is very simple. If the $\mathcal{N}=2$ half-BPS spectrum of K3 carries an action of the Mathieu group $\mathbb{M}_{24}$, then the same will apply to the symmetric power theories where $g \in \mathbb{M}_{24}$ acts diagonally on all $p$ factors so as to commute with the orbifold action. But if this is so, then we can also consider the twining character corresponding to $g \in \mathbb{M}_{24}$ for the $p^{\text {th }}$ symmetric power theory. Given our detailed understanding of the orbifold theory, we can determine how $g$ acts on the various terms in (2.17) or indeed on the different terms in $H_{p} \phi(\tau, z)$. The first term corresponds to the untwisted sector 
contribution where the cyclic permutation generator has been inserted into the trace. This implies that only the states of the form $(u \otimes u \otimes \cdots \otimes u)$ contribute. Inserting the diagonal group element $g \in \mathbb{M}_{24}$ then leads to $\phi_{g^{p}}(p \tau, p z)$. The other terms of $H_{p} \phi(\tau, z)$ come from the twisted sector, and the natural action of $g \in \mathbb{M}_{24}$ is simply described by replacing $\phi$ by $\phi_{g}$. Thus we are led to claim that the twining genus for the Hecke part of the $p^{\text {th }}$ symmetric power theory is given by

$$
\left(H_{p} \phi\right)_{g}(\tau, z)=\phi_{g^{p}}(p \tau, p z)+\sum_{l=0}^{p-1} \phi_{g}\left(\frac{\tau+l}{p}, z\right) .
$$

Translated into the NS-sector characters we then have for $p=2$

$$
\chi_{g}^{(2)}(\tau)=\chi_{g^{2}}\left(2 \tau, \frac{1}{2}\right)+\phi_{g}\left(\frac{\tau}{2}, \frac{1}{2}\right)+(-1)^{m} \phi_{g}\left(\frac{\tau}{2}+\frac{1}{2}, 0\right),
$$

while for $p>2$ the formula is

$$
\chi_{g}^{(p)}(\tau)=\chi_{g^{p}}(p \tau)+\sum_{l=0}^{p-1} \chi_{g}\left(\frac{\tau+4 l}{p}\right), \quad p \geq 3 \text { prime. }
$$

On the other hand, it is clear from the discussion around (3.12) that also these twining characters must be invariant under $\Gamma^{g}$, possibly up to a (different) multiplier system. In the context of Monstrous Moonshine, the McKay-Thompson series were Hauptmoduls for the corresponding genus zero congruence subgroups, and thus the McKay-Thompson series of the symmetric power theories could be written as a polynomial of the original McKayThompson series. This then leads to the replication formulae of Monstrous Moonshine (generalising directly the $J$-function replication identities of (2.23)).

In the current context, it appears that the twining characters are, on the face of it, not of genus zero, but we still find remarkable replication identities that give yet another consistency check on our analysis (and indicate that the twining characters corresponding to $3 \mathrm{~B}$ and $7 \mathrm{~A}=7 \mathrm{~B}$ have indeed good modular properties). Specifically, we have found the following identities for $p=2$

$$
\begin{aligned}
& \chi_{2 \mathrm{~A}}^{(2)}(\tau)=\chi_{1 \mathrm{~A}}\left(2 \tau, \frac{1}{2}\right)+\phi_{2 \mathrm{~A}}\left(\frac{\tau}{2}, \frac{1}{2}\right)-4=\chi_{2 \mathrm{~A}}(\tau)^{2}+4 \\
& \chi_{2 \mathrm{~B}}^{(2)}(\tau)=\chi_{1 \mathrm{~A}}\left(2 \tau, \frac{1}{2}\right)+\phi_{2 \mathrm{~B}}\left(\frac{\tau}{2}, \frac{1}{2}\right)=\chi_{2 \mathrm{~B}}(\tau)^{2}-12 \\
& \chi_{3 \mathrm{~A}}^{(2)}(\tau)=\chi_{3 \mathrm{~A}}\left(2 \tau, \frac{1}{2}\right)+\phi_{3 \mathrm{~A}}\left(\frac{\tau}{2}, \frac{1}{2}\right)-3=\chi_{3 \mathrm{~A}}(\tau)^{2} \\
& \chi_{3 \mathrm{~B}}^{(2)}(\tau)=\chi_{3 \mathrm{~B}}\left(2 \tau, \frac{1}{2}\right)+\phi_{3 \mathrm{~B}}\left(\frac{\tau}{2}, \frac{1}{2}\right)=-\frac{1}{2} \chi_{3 \mathrm{~B}}(\tau)^{2}+\frac{3}{2} T_{[12 \mathrm{D}]}\left(\frac{\tau}{2}\right) \\
& \chi_{4 \mathrm{~A}}^{(2)}(\tau)=\chi_{2 \mathrm{~A}}\left(2 \tau, \frac{1}{2}\right)+\phi_{4 \mathrm{~A}}\left(\frac{\tau}{2}, \frac{1}{2}\right)=-\chi_{4 \mathrm{~A}}(\tau)^{2}+2 T_{[8 \mathrm{E}]}\left(\frac{\tau}{2}\right)+4 \\
& \chi_{4 \mathrm{~B}}^{(2)}(\tau)=\chi_{2 \mathrm{~A}}\left(2 \tau, \frac{1}{2}\right)+\phi_{4 \mathrm{~B}}\left(\frac{\tau}{2}, \frac{1}{2}\right)-2=-\chi_{4 \mathrm{~B}}(\tau)^{2}+2 T_{[8 \mathrm{E}]}\left(\frac{\tau}{2}\right)-4
\end{aligned}
$$




$$
\begin{aligned}
& \chi_{4 \mathrm{C}}^{(2)}(\tau)=\chi_{2 \mathrm{~B}}\left(2 \tau, \frac{1}{2}\right)+\phi_{4 \mathrm{C}}\left(\frac{\tau}{2}, \frac{1}{2}\right)=-\frac{1}{2} \chi_{4 \mathrm{C}}(\tau)^{2}+\frac{3}{2} T_{[16 \mathrm{~d}]}\left(\frac{\tau}{4}\right)^{2} \\
& \chi_{5 \mathrm{~A}}^{(2)}(\tau)=\chi_{5 \mathrm{~A}}\left(2 \tau, \frac{1}{2}\right)+\phi_{5 \mathrm{~A}}\left(\frac{\tau}{2}, \frac{1}{2}\right)-2=-\chi_{5 \mathrm{~A}}(\tau)^{2}+2 T_{[20 \mathrm{C}]}\left(\frac{\tau}{2}\right)-4 \\
& \chi_{6 \mathrm{~A}}^{(2)}(\tau)=\chi_{3 \mathrm{~A}}\left(2 \tau, \frac{1}{2}\right)+\phi_{6 \mathrm{~A}}\left(\frac{\tau}{2}, \frac{1}{2}\right)-1=-\chi_{6 \mathrm{~A}}(\tau)^{2}+2 T_{[12 \mathrm{I}]}\left(\frac{\tau}{2}\right) \\
& \chi_{7 \mathrm{~A}}^{(2)}(\tau)=\chi_{7 \mathrm{~A}}\left(2 \tau, \frac{1}{2}\right)+\phi_{7 \mathrm{~A}}\left(\frac{\tau}{2}, \frac{1}{2}\right)-\frac{3}{2}=-2 \chi_{7 \mathrm{~A}}(\tau)^{2}+3 T_{[28 \mathrm{~B}]}\left(\frac{\tau}{2}\right) .
\end{aligned}
$$

These identities imply recursion relations for the twining characters which one could use, say, to determine the twining character for $3 \mathrm{~B}$ explicitly. Furthermore, since the McKayThompson series are known to have good modular transformation properties, the existence of these relations implies that the same must be true for the twining characters that appear here.

For $p=3$ we have found the following explicit identities

$$
\begin{aligned}
& \chi_{2 \mathrm{~A}}^{(3)}(\tau)=\chi_{2 \mathrm{~A}}(3 \tau)+\sum_{l=0}^{2} \chi_{2 \mathrm{~A}}\left(\frac{\tau+4 l}{3}\right)=\chi_{2 \mathrm{~A}}(\tau)^{3}+12 \chi_{2 \mathrm{~A}}(\tau) \\
& \chi_{2 \mathrm{~B}}^{(3)}(\tau)=\chi_{2 \mathrm{~B}}(3 \tau)+\sum_{l=0}^{2} \chi_{2 \mathrm{~B}}\left(\frac{\tau+4 l}{3}\right)=\chi_{2 \mathrm{~B}}(\tau)^{3}-12 \chi_{2 \mathrm{~B}}(\tau) \\
& \chi_{3 \mathrm{~A}}^{(3)}(\tau)=\chi_{1 \mathrm{~A}}(3 \tau)+\sum_{l=0}^{2} \chi_{3 \mathrm{~A}}\left(\frac{\tau+4 l}{3}\right)=\chi_{3 \mathrm{~A}}(\tau)^{3}+6 \chi_{3 \mathrm{~A}}(\tau) \\
& \chi_{4 \mathrm{~A}}^{(3)}(\tau)=\chi_{4 \mathrm{~A}}(3 \tau)+\sum_{l=0}^{2} \chi_{4 \mathrm{~A}}\left(\frac{\tau+4 l}{3}\right)=\chi_{4 \mathrm{~A}}(\tau)^{3}-12 \chi_{4 \mathrm{~A}}(\tau) \\
& \chi_{4 \mathrm{~B}}^{(3)}(\tau)=\chi_{4 \mathrm{~B}}(3 \tau)+\sum_{l=0}^{2} \chi_{4 \mathrm{~B}}\left(\frac{\tau+4 l}{3}\right)=\chi_{4 \mathrm{~B}}(\tau)^{3} \\
& \chi_{4 \mathrm{C}}^{(3)}(\tau)=\chi_{4 \mathrm{C}}(3 \tau)+\sum_{l=0}^{2} \chi_{4 \mathrm{C}}\left(\frac{\tau+4 l}{3}\right)=\frac{1}{4} \chi_{4 \mathrm{C}}(\tau)^{3}+\frac{3}{4} \chi_{4 \mathrm{C}}(\tau) T_{[16 \mathrm{~d}]}\left(\frac{\tau}{4}\right)^{2}-6 T_{[16 \mathrm{~d}]}\left(\frac{\tau}{4}\right) \\
& \chi_{5 \mathrm{~A}}^{(3)}(\tau)=\chi_{5 \mathrm{~A}}(3 \tau)+\sum_{l=0}^{2} \chi_{5 \mathrm{~A}}\left(\frac{\tau+4 l}{3}\right)=\chi_{5 \mathrm{~A}}(\tau)^{3} \\
& \chi_{6 \mathrm{~A}}^{(3)}(\tau)=\chi_{2 \mathrm{~A}}(3 \tau)+\sum_{l=0}^{2} \chi_{6 \mathrm{~A}}\left(\frac{\tau+4 l}{3}\right)=\chi_{6 \mathrm{~A}}(\tau)^{3}-6 \chi_{6 \mathrm{~A}}(\tau)
\end{aligned}
$$

while for $p=5$ we have found

$$
\chi_{2 \mathrm{~A}}^{(5)}(\tau)=\chi_{2 \mathrm{~A}}(5 \tau)+\sum_{l=0}^{4} \chi_{2 \mathrm{~A}}\left(\frac{\tau+4 l}{5}\right)=\chi_{2 \mathrm{~A}}(\tau)^{5}+20 \chi_{2 \mathrm{~A}}(\tau)^{3}+70 \chi_{2 \mathrm{~A}}(\tau)
$$




$$
\begin{aligned}
\chi_{2 \mathrm{~B}}^{(5)}(\tau) & =\chi_{2 \mathrm{~B}}(5 \tau)+\sum_{l=0}^{4} \chi_{2 \mathrm{~B}}\left(\frac{\tau+4 l}{5}\right)=\chi_{2 \mathrm{~B}}(\tau)^{5}-20 \chi_{2 \mathrm{~B}}(\tau)^{3}+70 \chi_{2 \mathrm{~B}}(\tau) \\
\chi_{3 \mathrm{~A}}^{(5)}(\tau) & =\chi_{3 \mathrm{~A}}(5 \tau)+\sum_{l=0}^{4} \chi_{3 \mathrm{~A}}\left(\frac{\tau+4 l}{5}\right)=\chi_{3 \mathrm{~A}}(\tau)^{5}+10 \chi_{3 \mathrm{~A}}(\tau)^{3}+15 \chi_{3 \mathrm{~A}}(\tau) \\
\chi_{4 \mathrm{~A}}^{(5)}(\tau)=\chi_{4 \mathrm{~A}}(5 \tau)+\sum_{l=0}^{4} \chi_{4 \mathrm{~A}}\left(\frac{\tau+4 l}{5}\right) & =\chi_{4 \mathrm{~A}}(\tau)^{5}-20 \chi_{4 \mathrm{~A}}(\tau)^{3}+30 \chi_{4 \mathrm{~A}}(\tau) \\
\chi_{4 \mathrm{~B}}^{(5)}(\tau)=\chi_{4 \mathrm{~B}}(5 \tau)+\sum_{l=0}^{4} \chi_{4 \mathrm{~B}}\left(\frac{\tau+4 l}{5}\right) & =\chi_{4 \mathrm{~B}}(\tau)^{5}-10 \chi_{4 \mathrm{~B}}(\tau) \\
\chi_{4 \mathrm{C}}^{(5)}(\tau)=\chi_{4 \mathrm{C}}(5 \tau)+\sum_{l=0}^{4} \chi_{4 \mathrm{C}}\left(\frac{\tau+4 l}{5}\right) & =\frac{1}{16} \chi_{4 \mathrm{C}}(\tau)^{5}-\frac{1}{16} \chi_{4 \mathrm{C}}(\tau)^{3} T_{[16 \mathrm{~d}]}\left(\frac{\tau}{4}\right)^{2} \\
& \chi_{[16 \mathrm{~d}]}\left(\frac{\tau}{4}\right)^{4}-\frac{1}{2} \chi_{4 \mathrm{C}}(\tau)^{2} T_{[16 \mathrm{~d}]}\left(\frac{\tau}{4}\right)-4 T_{[16 \mathrm{~d}]}\left(\frac{\tau}{4}\right)^{3}-2 \chi_{4 \mathrm{C}}(\tau) \\
\chi_{5 \mathrm{~A}}^{(5)}(\tau)=\chi_{1 \mathrm{~A}}(5 \tau)+\sum_{l=0}^{4} \chi_{5 \mathrm{~A}}\left(\frac{\tau+4 l}{5}\right) & =\chi_{5 \mathrm{~A}}(\tau)^{5}-15 \chi_{5 \mathrm{~A}}(\tau) \\
\chi_{6 \mathrm{~A}}^{(5)}(\tau)=\chi_{6 \mathrm{~A}}(5 \tau)+\sum_{l=0}^{4} \chi_{6 \mathrm{~A}}\left(\frac{\tau+4 l}{5}\right) & =\chi_{6 \mathrm{~A}}(\tau)^{5}-10 \chi_{6 \mathrm{~A}}(\tau)^{3}-5 \chi_{6 \mathrm{~A}}(\tau)
\end{aligned}
$$

These are probably not the only identities that exist, but only those we have been able to find with our current partial understanding of the twining characters. Indeed, in order to be able to determine the higher order replication formulae, one needs to know the twining characters up to some fairly high level. Thus with our current understanding we can only check these identities for the twining characters given in (3.14).

\section{Conclusions}

In this paper we have provided strong evidence for the conjecture of Eguchi, Ooguri and Tachikawa [11] that the elliptic genus of K3 carries a natural action of the Mathieu group $\mathbb{M}_{24}$. In particular, we have shown that the elliptic genus with the insertion of a group element in $\mathbb{M}_{24}$ leads, in the NS-sector, to a twining character that possesses nice modular properties. By considering the twining characters of symmetric power theories we have furthermore shown that the twining characters satisfy beautiful replication identities similar to those appearing for the McKay-Thompson series in Monstrous Moonshine. While the analogy of the twining characters with the McKay-Thompson series is quite striking, there are also marked differences: at least on the face of it, the twining characters are not Hauptmoduls for a genus zero congruence subgroup since their replication relations contain also 'inhomogeneous' terms (for example the terms proportional to $T_{[16 \mathrm{~d}]}$ in (3.34) and (3.42)). 
So far we have only worked out about half of the twining characters in detail. In order to get a more complete understanding of the Mathieu action on the K3 elliptic genus, it would be very desirable to find closed form expressions for all twining characters. Probably some more refined techniques (rather than just guessing and confirming the answer) will be necessary; in particular, the techniques developed by Bantay \& Gannon [1, 2] should be useful for this.

The above analysis suggests that the space of $\mathcal{N}=2$ half-BPS states of K3 carries an action of the Mathieu group $\mathbb{M}_{24}$. It is believed that these $\mathcal{N}=2$ half-BPS states form a subset of the quarter-BPS states (with respect to $\mathcal{N}=4$ ), for which the spectrum generating algebra is the so-called BPS-algebra of Harvey \& Moore [7, 19, 20]. The above group action on the spectrum then suggests that the Mathieu group $\mathbb{M}_{24}$ should be (contained in) the automorphism group of that Borcherds algebra, in complete analogy to what happened for Monstrous Moonshine. It would be very interesting to explore this idea in more detail.

\section{Acknowledgments}

We thank Terry Gannon for useful communications and Daniel Persson for comments on the draft. The research of MRG is partially supported by a grant from the Swiss National Science Foundation, and the research of RV is supported by an INFN Fellowship.

Note added. As this paper was being finalised, a preprint appeared arXiv : 1005 . 5415 [4] that contains overlapping results with those of section 3.1 of the present paper.

\section{A Definitions}

Our conventions for the Dedekind eta and the Jacobi theta functions are

$$
\begin{aligned}
\eta(\tau) & =q^{\frac{1}{24}} \prod_{n=1}^{\infty}\left(1-q^{n}\right) \\
\vartheta_{1}(\tau, z) & =-i q^{\frac{1}{8}} y^{\frac{1}{2}} \prod_{n=1}^{\infty}\left(1-q^{n}\right)\left(1-y q^{n}\right)\left(1-y^{-1} q^{n-1}\right) \\
\vartheta_{2}(\tau, z) & =2 q^{\frac{1}{8}} \cos (\pi z) \prod_{n=1}^{\infty}\left(1-q^{n}\right)\left(1+y q^{n}\right)\left(1+y^{-1} q^{n}\right) \\
\vartheta_{3}(\tau, z) & =\prod_{n=1}^{\infty}\left(1-q^{n}\right)\left(1+y q^{n-1 / 2}\right)\left(1+y^{-1} q^{n-1 / 2}\right) \\
\vartheta_{4}(\tau, z) & =\prod_{n=1}^{\infty}\left(1-q^{n}\right)\left(1-y q^{n-1 / 2}\right)\left(1-y^{-1} q^{n-1 / 2}\right) .
\end{aligned}
$$

Under modular transformations the $\vartheta$ and $\eta$ functions transform as

$$
\begin{aligned}
\eta(\tau+1) & =e^{\frac{2 \pi i}{24}} \eta(\tau) & \vartheta_{2}(\tau+1, z) & =e^{\frac{2 \pi i}{8}} \vartheta_{2}(\tau, z), \\
\vartheta_{3}(\tau+1, z) & =\vartheta_{4}(\tau, z) & \vartheta_{4}(\tau+1, z) & =\vartheta_{3}(\tau, z),
\end{aligned}
$$


as well as

$$
\begin{array}{cl}
\eta\left(-\frac{1}{\tau}\right)=(-i \tau)^{\frac{1}{2}} \eta(\tau) & \vartheta_{2}\left(-\frac{1}{\tau}, \frac{z}{\tau}\right)=(-i \tau)^{\frac{1}{2}} e^{\frac{i \pi z^{2}}{\tau}} \vartheta_{4}(\tau, z), \\
\vartheta_{3}\left(-\frac{1}{\tau}, \frac{z}{\tau}\right)=(-i \tau)^{\frac{1}{2}} e^{\frac{i \pi z^{2}}{\tau}} \vartheta_{3}(\tau, z) & \vartheta_{4}\left(-\frac{1}{\tau}, \frac{z}{\tau}\right)=(-i \tau)^{\frac{1}{2}} e^{\frac{i \pi z^{2}}{\tau}} \vartheta_{2}(\tau, z) .
\end{array}
$$

The theta functions $\vartheta_{a}(\tau)$ are defined as $\vartheta_{a}(\tau) \equiv \vartheta_{a}(\tau, z=0)$.

\section{A.1 McKay-Thompson series}

For completeness we give here the first few terms of the McKay-Thompson series that appear in our analysis

$$
\begin{aligned}
& T_{[8 \mathrm{D}]}=q^{-1}+4 q+2 q^{3}+8 q^{5}-q^{7}+\cdots \\
& T_{[8 \mathrm{E}]}=q^{-1}+4 q+2 q^{3}-8 q^{5}-q^{7}+\cdots \\
& T_{[8 \mathrm{a}]}=q^{-1}-20 q-62 q^{3}-216 q^{5}-641 q^{7}-1636 q^{9}-\cdots \\
& T_{[12 \mathrm{D}]}=q^{-1}+8 q^{2}+28 q^{5}+64 q^{8}+134 q^{11}+\cdots \\
& T_{[12 \mathrm{I}]}=q^{-1}+2 q+q^{3}-2 q^{7}-2 q^{9}+2 q^{11}+\cdots \\
& T_{[16 \mathrm{~A}]}=q^{-1}+4 q+10 q^{3}+24 q^{5}+47 q^{7}+84 q^{9}+\cdots \\
& T_{[16 \mathrm{~B}]}=q^{-1}+2 q^{3}-q^{7}-2 q^{11}+3 q^{15}+2 q^{19}+\cdots \\
& T_{[16 \mathrm{~b}]}=q^{-1}+4 q-2 q^{3}+8 q^{5}-q^{7}+20 q^{9}+\cdots \\
& T_{[16 \mathrm{~d}]}=T_{[8 \mathrm{D}]}(2 \tau)^{1 / 2}=q^{-1}-2 q^{3}-q^{7}+2 q^{11}+\cdots \\
& T_{[20 \mathrm{C}]}=q^{-1}+q-2 q^{2}+2 q^{3}+2 q^{4}-q^{5}-4 q^{7}+\cdots \\
& T_{[24 \mathrm{H}]}=q^{-1}+2 q+5 q^{3}+8 q^{5}+14 q^{7}+22 q^{9}+\cdots \\
& T_{[24 \mathrm{c}]}=q^{-1}-2 q+q^{3}-2 q^{7}+2 q^{9}+2 q^{11}+\cdots \\
& T_{[28 \mathrm{~B}]}=q^{-1}+3 q+4 q^{2}+9 q^{3}+12 q^{4}+15 q^{5}+24 q^{6}+39 q^{7}+\cdots \\
& T_{[40 \mathrm{a}]}=q^{-1}+3 q^{3}+4 q^{5}+4 q^{7}+4 q^{9}+7 q^{11}+\cdots,
\end{aligned}
$$

see [15] and http://www.research.att.com/ njas/sequences/for more information about these series.

\section{A.2 Twining characters for $\mathrm{K} 3$}

Here we give the low order expansion of the remaining twining characters. Since the spectrum of the BPS states is real, the twining characters of conjugacy classes that only differ on conjugate representations agree. The remaining functions are therefore

$$
\begin{gathered}
\chi_{3 \mathrm{~B}}(\tau)=q^{-\frac{1}{4}}\left(1+4 q^{\frac{1}{2}}+4 q+4 q^{2}+8 q^{\frac{5}{2}}+2 q^{3}+8 q^{\frac{7}{2}}+12 q^{4}+4 q^{\frac{9}{2}}+16 q^{5}+16 q^{\frac{11}{2}}\right. \\
\left.+5 q^{6}+26 q^{\frac{13}{2}}+21 q^{7}+\cdots\right) \\
\chi_{6 \mathrm{~B}}(\tau)=q^{-\frac{1}{4}}\left(1+4 q^{\frac{1}{2}}+8 q+16 q^{\frac{3}{2}}+32 q^{2}+56 q^{\frac{5}{2}}+94 q^{3}++152 q^{\frac{7}{2}}+240 q^{4}+372 q^{\frac{9}{2}}\right. \\
\left.+560 q^{5}+832 q^{\frac{11}{2}}+1197 q^{6}+1594 q^{\frac{13}{2}}+1849 q^{7}+\cdots\right)
\end{gathered}
$$




$$
\begin{aligned}
& \chi_{7 \mathrm{~A}}(\tau)=\chi_{7 \mathrm{~B}}(\tau)=q^{-\frac{1}{4}}\left(1+q^{\frac{1}{2}}+\frac{9}{2} q+8 q^{\frac{3}{2}}+\frac{27}{2} q^{2}+23 q^{\frac{5}{2}}+\frac{81}{2} q^{3}+68 q^{\frac{7}{2}}+104 q^{4}\right. \\
& \left.+154 q^{\frac{9}{2}}+\frac{469}{2} q^{5}+352 q^{\frac{11}{2}}+\frac{987}{2} q^{6}+\frac{1293}{2} q^{\frac{13}{2}}+751 q^{7}+\cdots\right) \\
& \chi_{8 \mathrm{~A}}(\tau)=q^{-\frac{1}{4}}\left(1+2 q^{\frac{1}{2}}+6 q+12 q^{\frac{3}{2}}+23 q^{2}+42 q^{\frac{5}{2}}+74 q^{3}+124 q^{\frac{7}{2}}+203 q^{4}+324 q^{\frac{9}{2}}\right. \\
& \left.+502 q^{5}+768 q^{\frac{11}{2}}+1141 q^{6}+1567 q^{\frac{13}{2}}+1866 q^{7}+\cdots\right) \\
& \chi_{10 \mathrm{~A}}(\tau)=q^{-\frac{1}{4}}\left(1+4 q^{\frac{1}{2}}+7 q+12 q^{\frac{3}{2}}+24 q^{2}+40 q^{\frac{5}{2}}+63 q^{3}+100 q^{\frac{7}{2}}+153 q^{4}+232 q^{\frac{9}{2}}\right. \\
& \left.+342 q^{5}+492 q^{\frac{11}{2}}+692 q^{6}+900 q^{\frac{13}{2}}+1018 q^{7}+\cdots\right) \\
& \chi_{11 \mathrm{~A}}(\tau)=q^{-\frac{1}{4}}\left(1+2 q^{\frac{1}{2}}+4 q+4 q^{\frac{3}{2}}+8 q^{2}+14 q^{\frac{5}{2}}+17 q^{3}+24 q^{\frac{7}{2}}+37 q^{4}+52 q^{\frac{9}{2}}\right. \\
& \left.+68 q^{5}+88 q^{\frac{11}{2}}+116 q^{6}+141 q^{\frac{13}{2}}+147 q^{7}+\cdots\right) \\
& \chi_{12 \mathrm{~A}}(\tau)=q^{-\frac{1}{4}}\left(1+4 q^{\frac{1}{2}}+7 q+12 q^{\frac{3}{2}}+26 q^{2}+48 q^{\frac{5}{2}}+78 q^{3}+128 q^{\frac{7}{2}}+211 q^{4}+336 q^{\frac{9}{2}}\right. \\
& \left.+516 q^{5}+780 q^{\frac{11}{2}}+1157 q^{6}+1592 q^{\frac{13}{2}}+1886 q^{7}+\cdots\right) \\
& \chi_{14 \mathrm{~A}}(\tau)=\chi_{14 \mathrm{~B}}(\tau)=q^{-\frac{1}{4}}\left(1+3 q^{\frac{1}{2}}+\frac{11}{2} q+8 q^{\frac{3}{2}}+\frac{33}{2} q^{2}+29 q^{\frac{5}{2}}+\frac{87}{2} q^{3}+68 q^{\frac{7}{2}}+108 q^{4}\right. \\
& \left.+166 q^{\frac{9}{2}}+\frac{487}{2} q^{5}+352 q^{\frac{11}{2}}+\frac{1009}{2} q^{6}+\frac{1335}{2} q^{\frac{13}{2}}+761 q^{7}+\cdots\right) \\
& \chi_{15 \mathrm{~A}}(\tau)=\chi_{15 \mathrm{~B}}(\tau)=q^{-\frac{1}{4}}\left(1+3 q^{\frac{1}{2}}+6 q+10 q^{\frac{3}{2}}+\frac{41}{2} q^{2}+37 q^{\frac{5}{2}}+\frac{119}{2} q^{3}+96 q^{\frac{7}{2}}+\frac{311}{2} q^{4}\right. \\
& \left.+244 q^{\frac{9}{2}}+367 q^{5}+544 q^{\frac{11}{2}}+795 q^{6}+\frac{2149}{2} q^{\frac{13}{2}}+1250 q^{7}+\cdots\right) \\
& \chi_{21 \mathrm{~A}}(\tau)=\chi_{21 \mathrm{~B}}(\tau)=q^{-\frac{1}{4}}\left(1+4 q^{\frac{1}{2}}+\frac{15}{2} q+14 q^{\frac{3}{2}}+\frac{57}{2} q^{2}+50 q^{\frac{5}{2}}+\frac{165}{2} q^{3}+134 q^{\frac{7}{2}}+215 q^{4}\right. \\
& \left.+340 q^{\frac{9}{2}}+\frac{1033}{2} q^{5}+772 q^{\frac{11}{2}}+\frac{2271}{2} q^{6}+1545 q^{\frac{13}{2}}+1813 q^{7}+\cdots\right) \\
& \chi_{23 \mathrm{~A}}(\tau)=\chi_{23 \mathrm{~B}}(\tau)=q^{-\frac{1}{4}}\left(1+3 q^{\frac{1}{2}}+7 q+14 q^{\frac{3}{2}}+26 q^{2}+43 q^{\frac{5}{2}}+\frac{149}{2} q^{3}+124 q^{\frac{7}{2}}+\frac{383}{2} q^{4}\right. \\
& \left.+296 q^{\frac{9}{2}}+\frac{909}{2} q^{5}+682 q^{\frac{11}{2}}+985 q^{6}+\frac{2639}{2} q^{\frac{13}{2}}+1548 q^{7}+\cdots\right) .
\end{aligned}
$$




\begin{tabular}{|c|c|c|c|c|c|c|c|c|c|c|c|c|c|c|c|c|c|c|c|c|c|c|c|c|c|}
\hline 1 & 2 & 3 & & 4 & 5 & 6 & 7 & 8 & 9 & 10 & 11 & 12 & 13 & 14 & 15 & 16 & 17 & 18 & 19 & 20 & 21 & 22 & 23 & 2425 & 26 \\
\hline $1 \mathrm{~A}$ & $2 \mathrm{~A}$ & A & $5 \mathrm{~A}$ & A $4 \mathrm{I}$ & $B$ & $7 \mathrm{~A}$ & $7 \mathrm{~B}$ & $8 \mathrm{~A}$ & $6 \mathrm{~A}$ & $11 \mathrm{~A}$ & $15 \mathrm{~A}$ & $15 \mathrm{~B}$ & $14 \mathrm{~A}$ & $14 \mathrm{~B}$ & $23 \mathrm{~A}$ & $23 \mathrm{~B}$ & $12 \mathrm{~B}$ & $6 \mathrm{~B}$ & $4 \mathrm{C}$ & $3 \mathrm{~B}$ & $2 \mathrm{~B}$ & $10 \mathrm{~A}$ & $21 \mathrm{~A}$ & $21 \mathrm{~B} 4 \mathrm{~A}$ & $12 \mathrm{~A}$ \\
\hline & & & & & $\mathrm{A}$ & & & & $\begin{array}{l}3 \mathrm{AA} \\
2 \mathrm{~A}\end{array}$ & & $3 \mathrm{~A}$ & $3 \mathrm{~A}$ & $2 \mathrm{~A}$ & $2 \mathrm{~A}$ & & & & & $2 \mathrm{~B}$ & & & $2 \mathrm{~B}$ & $3 \mathrm{~B}$ & $3 \mathrm{~B} 2 \mathrm{~A}$ & $\begin{array}{l}6 \mathrm{~A} \\
4 \mathrm{~A}\end{array}$ \\
\hline 1 & 1 & 1 & & 1 & 1 & 1 & 1 & 1 & 1 & 1 & 1 & 1 & 1 & 1 & 1 & 1 & 1 & 1 & 1 & 1 & 1 & 1 & 1 & 1 & 1 \\
\hline 23 & 7 & 5 & & 3 & 3 & 2 & 2 & 1 & 1 & 1 & 0 & 0 & 0 & 0 & 0 & 0 & -1 & -1 & -1 & -1 & -1 & -1 & -1 & $-1-1$ & -1 \\
\hline 252 & 28 & 9 & & 2 & 4 & 0 & 0 & 0 & 1 & -1 & -1 & -1 & 0 & 0 & -1 & -1 & 0 & 0 & 0 & 0 & 12 & 2 & 0 & $0 \quad 4$ & 1 \\
\hline 253 & 13 & 10 & & 3 & 1 & 1 & & -1 & -2 & 0 & 0 & 0 & -1 & -1 & 0 & 0 & 1 & 1 & 1 & & -11 & -1 & 1 & $1-3$ & 0 \\
\hline 1771 & -21 & 16 & & $1-$ & & 0 & & -1 & 0 & 0 & 1 & 1 & 0 & 0 & 0 & 0 & -1 & -1 & -1 & 7 & 11 & 1 & 0 & $0 \quad 3$ & 0 \\
\hline 3520 & 64 & 10 & & 0 & 0 & -1 & -1 & & -2 & 0 & 0 & 0 & 1 & 1 & 1 & 1 & 0 & 0 & 0 & -8 & 0 & 0 & -1 & $\begin{array}{ll}-1 & 0\end{array}$ & 0 \\
\hline 45 & -3 & 0 & & 0 & 1 & $e_{7}^{+}$ & $e_{7}^{-}$ & -1 & 0 & 1 & 0 & 0 & $-e_{7}^{+}$ & $-e_{7}^{-}$ & -1 & -1 & & -1 & 1 & 3 & 5 & 0 & $e_{7}^{-}$ & $e_{7}^{+}-3$ & 0 \\
\hline 45 & -3 & 0 & & 0 & 1 & $e_{7}^{-\frac{-}{2}}$ & $e_{7}^{+}$ & -1 & 0 & 1 & 0 & 0 & $-e^{\frac{-}{7}}$ & $-e_{7}^{+}$ & -1 & -1 & & -1 & 1 & 3 & 5 & 0 & $e_{7}^{+}$ & $e^{\frac{-}{7}}-3$ & 0 \\
\hline 990 & -18 & 0 & & 0 & & $e_{7}^{+}$ & $e_{7}^{-}$ & 0 & 0 & 0 & 0 & 0 & $e_{7}^{+}$ & $e_{7}^{-}$ & 1 & 1 & & -1 & -2 & 3 & -10 & 0 & $e_{7}^{-}$ & $e_{7}^{+}$ & 0 \\
\hline 990 & -18 & 0 & & 0 & & $e_{\overline{7}}^{-\overline{-}}$ & $e_{7}^{+}$ & 0 & 0 & 0 & 0 & 0 & $e_{7}^{-}$ & $e_{7}^{+}$ & 1 & 1 & & -1 & -2 & & -10 & 0 & $e_{7}^{+}$ & $e_{\overline{7}}^{\dot{-}}$ & 0 \\
\hline 1035 & -21 & 0 & & 0 & & $2 e_{7}^{+}$ & $2 e_{\overline{7}}^{-}$ & -1 & 0 & 1 & 0 & 0 & 0 & 0 & 0 & 0 & -1 & & -1 & -3 & -5 & & $-e_{7}^{-}$ & $-e_{7}^{+}$ & 0 \\
\hline 1035 & -21 & 0 & & 0 & & $2 e_{7}^{-}$ & $2 e_{7}^{+}$ & -1 & 0 & 1 & 0 & 0 & 0 & 0 & 0 & 0 & -1 & 1 & -1 & -3 & -5 & 0 & $-e_{7}^{+}$ & $-e_{7}^{-}$ & 0 \\
\hline 1035 & 27 & 0 & & $0-$ & -1 & -1 & -1 & 1 & 0 & 1 & 0 & 0 & -1 & -1 & 0 & 0 & 0 & 2 & 3 & 6 & 35 & 0 & -1 & -1 & 0 \\
\hline 231 & 7 & -3 & & $1-$ & & 0 & & -1 & 1 & 0 & $e_{15}^{+}$ & $e_{15}^{-}$ & 0 & 0 & 1 & 1 & 0 & 0 & 3 & 0 & -9 & 1 & 0 & $0-1$ & -1 \\
\hline 231 & 7 & -3 & & $1-$ & & 0 & & -1 & 1 & 0 & $e_{15}^{-}$ & $e_{15}^{+}$ & 0 & 0 & 1 & 1 & 0 & 0 & 3 & 0 & -9 & 1 & 0 & $0-1$ & -1 \\
\hline 770 & -14 & 5 & & $0-$ & & 0 & 0 & & 1 & 0 & 0 & 0 & 0 & 0 & $e_{23}^{+}$ & $e_{23}^{-}$ & 1 & 1 & -2 & -7 & 10 & 0 & 0 & 0 & -1 \\
\hline 770 & -14 & 5 & & $0-$ & & 0 & 0 & & 1 & 0 & 0 & 0 & 0 & 0 & $e_{23}^{-}$ & $e_{23}^{+}$ & 1 & 1 & -2 & -7 & 10 & 0 & 0 & 0 & -1 \\
\hline 483 & 35 & 6 & -2 & & 3 & 0 & & -1 & 2 & -1 & 1 & 1 & 0 & 0 & 0 & 0 & 0 & 0 & 3 & 0 & 3 & -2 & 0 & 0 & 0 \\
\hline 1265 & 49 & 5 & & 0 & 1 & -2 & -2 & 1 & 1 & 0 & 0 & 0 & 0 & 0 & 0 & 0 & 0 & & -3 & & -15 & 0 & 1 & $1-7$ & -1 \\
\hline 2024 & 8 & -1 & -1 & & 0 & 1 & 1 & & -1 & 0 & -1 & -1 & 1 & 1 & 0 & 0 & 0 & 0 & 0 & 8 & 24 & -1 & 1 & 1 & -1 \\
\hline 2277 & 21 & & -3 & & 1 & 2 & & -1 & 0 & 0 & 0 & 0 & 0 & 0 & 0 & 0 & 0 & & -3 & & -19 & 1 & -1 & $-1-3$ & 0 \\
\hline 3312 & 48 & & -3 & & 0 & 1 & 1 & & 0 & 1 & 0 & 0 & -1 & -1 & 0 & 0 & & -2 & & -6 & 16 & 1 & 1 & 10 & 0 \\
\hline 5313 & 49 & -15 & & $3-$ & -3 & 0 & & -1 & 1 & 0 & 0 & 0 & 0 & 0 & 0 & 0 & 0 & & -3 & 0 & 9 & -1 & 0 & 0 & 1 \\
\hline 5796 & -28 & -9 & & 1 & 4 & 0 & 0 & 0 & -1 & -1 & 1 & 1 & 0 & 0 & 0 & 0 & 0 & 0 & 0 & 0 & 36 & 1 & 0 & $0-4$ & -1 \\
\hline 5544 & -56 & & -1 & & 0 & 0 & 0 & 0 & ) 1 & 0 & -1 & -1 & 0 & 0 & 1 & 1 & 0 & 0 & 0 & 0 & 24 & -1 & 0 & $0-8$ & 1 \\
\hline 10395 & -21 & 0 & & $0-$ & -1 & 0 & 0 & 1 & 0 & 0 & 0 & 0 & 0 & 0 & -1 & -1 & 0 & 0 & 3 & & -45 & 0 & 0 & 0 & \\
\hline
\end{tabular}

Table 1. The character table of the Mathieu group. The rows correspond to the characters, while the columns describe the different conjugacy classes, numbered from 1 to 26 as in [11]. The second line gives the name of the conjugacy class that is used throughout the paper, where the number is the order of any element in the class. Where appropriate the classes of some powers are given in the third and fourth row, but only the non-trivial cases are stated. Finally, $e_{p}^{ \pm}=(-1 \pm i \sqrt{p}) / 2$. 


\section{References}

[1] P. Bantay and T. Gannon, Conformal characters and the modular representation, JHEP 02 (2006) 005 [hep-th/0512011] [SPIRES].

[2] P. Bantay and T. Gannon, Vector-valued modular functions for the modular group and the hypergeometric equation, Commun. Number Theor. Phys. 1 (2008) 637 [arXiv:0705.2467].

[3] R. Borcherds, Vertex algebras, Kac-Moody algebras and the Monster, Proc. Nat. Acad. Sci. U.S.A. 83 (1986) 3068.

[4] M.C.N. Cheng, K3 surfaces, $N=4$ dyons and the Mathieu group M24, arXiv:1005.5415 [SPIRES].

[5] J.H. Conway and S. Norton, Monstrous moonshine, Bull. Lond. Math. Soc. 11 (1979) 308.

[6] R. Dijkgraaf, G.W. Moore, E.P. Verlinde and H.L. Verlinde, Elliptic genera of symmetric products and second quantized strings, Commun. Math. Phys. 185 (1997) 197 [hep-th/9608096] [SPIRES].

[7] R. Dijkgraaf, E.P. Verlinde and H.L. Verlinde, Counting dyons in $N=4$ string theory, Nucl. Phys. B 484 (1997) 543 [hep-th/9607026] [SPIRES].

[8] C.Y. Dong and G. Mason, An Orbifold theory of genus zero associated to the sporadic group $M_{24}$, Commun. Math. Phys. 164 (1994) 87 [SPIRES].

[9] T. Eguchi and K. Hikami, Superconformal algebras and Mock theta functions 2. Rademacher expansion for K3 surface, arXiv:0904.0911 [SPIRES].

[10] T. Eguchi, H. Ooguri, A. Taormina and S.-K. Yang, Superconformal algebras and string compactification on manifolds with $\mathrm{SU}(N)$ holonomy, Nucl. Phys. B 315 (1989) 193 [SPIRES].

[11] T. Eguchi, H. Ooguri and Y. Tachikawa, Notes on the K3 surface and the Mathieu group $M_{24}$, arXiv: 1004.0956 [SPIRES].

[12] T. Eguchi and A. Taormina, Character formulas for the $N=4$ superconformal algebra, Phys. Lett. B 200 (1988) 315 [SPIRES].

[13] T. Eguchi and A. Taormina, On the unitary representations of $N=2$ and $N=4$ superconformal algebras, Phys. Lett. B 210 (1988) 125 [SPIRES].

[14] M. Eichler and D. Zagier, The theory of Jacobi forms, Birkhäuser Germany (1985).

[15] D. Ford, J. McKay and S. Norton, More on replicable functions, Commun. Algebra 22 (1994) 5175 .

[16] I. Frenkel, J. Lepowski and A. Meurman, Vertex operator algebras and the Monster, Academic Press U.S.A. (1986).

[17] M.R. Gaberdiel, S. Gukov, C.A. Keller, G.W. Moore and H. Ooguri, Extremal N = $(2,2) 2 D$ conformal field theories and constraints of modularity, arXiv:0805.4216 [SPIRES].

[18] T. Gannon, Moonshine beyond the Monster: the bridge connecting algebra, modular forms and physics, Cambridge University Press, Cambridge U.K. (2006).

[19] J.A. Harvey and G.W. Moore, Algebras, BPS states and strings, Nucl. Phys. B 463 (1996) 315 [hep-th/9510182] [SPIRES].

[20] J.A. Harvey and G.W. Moore, On the algebras of BPS states, Commun. Math. Phys. 197 (1998) 489 [hep-th/9609017] [SPIRES]. 
[21] T. Kawai, Y. Yamada and S.-K. Yang, Elliptic genera and $N=2$ superconformal field theory, Nucl. Phys. B 414 (1994) 191 [hep-th/9306096] [SPIRES].

[22] J.G. Thompson, Some numerology between the Fischer-Griess Monster and the elliptic modular function, Bull. Lond. Math. Soc. 11 (1979) 352.

[23] M.P. Tuite, Monstrous moonshine from orbifolds, Commun. Math. Phys. 146 (1992) 277 [SPIRES]. 